\title{
PROPAGATION OF TORSIONAL SURFACE WAVES IN A NON- HOMOGENEOUS HALF-SPACE WITH CIRCULAR IRREGULARITY IN FREE SURFACE
}

\author{
M. SETHI* \\ Govt. Polytechnic, Faculty of Science \\ Jalandhar, INDIA \\ E-mail: munish_sethi26@yahoo.co.in; munishsethi76@gmail.com \\ A.K. SHARMA \\ Chandigarh Engg. College, Faculty of Applied Sciences \\ Chandigarh, INDIA \\ A. SHARMA \\ NIPER, Deputy Registrar, U.P. \\ INDIA
}

\begin{abstract}
The present paper studies the effect of circular regularity on propagation of torsional surface waves in an elastic non-homogeneous half-space. Both rigidity and density of the half-space are assumed to vary inversely linearly with depth. Separation of variable method has been used to get the analytical solutions for the dispersion equation of the torsional surface waves. Also, the effects of non-homogeneity and irregularity on the phase velocity of torsional surface waves have shown graphically.
\end{abstract}

Key words: non-homogeneous media, torsional waves, rigidity, irregularity.

\section{Introduction}

The formulations and solutions of many problems of linear wave-propagation for homogeneous media are available in the literature of continuum mechanics of solids. In recent years, however, sufficient interest has arisen in the problem connected with bodies whose mechanical properties are functions of space, i.e. non-homogeneous bodies. This interest is mainly due to the advent of solid rocket propellants, polymeric materials and growing demand of engineering and industrial applications. Studies of wave propagation in the earth stratum under loads have usually assumed that the earth behaves to a first approximation as an ideal elastic or visco-elastic material. The strata may be of finite depth or it may be so deep compared to the size of the loaded area that it can be regarded as a half-space. In either case the complete solutions to elastic or viscoelastic problems are known when material parameters are treated independent of position.

The formation of earth strata in nature tends, however, to result in depth variations of these parameters and this may be due principally, either to stratification of different materials or to the effect of superincumbent pressure. The case of homogeneous viscoelastic half-space has been treated extensively in the literature. By comparison little has been done on the non-homogenous viscoelastic half-space when the material characteristics vary continuously with depth. This fact is likely to be true when the effects of over-

\footnotetext{
* To whom correspondence should be addressed
} 
burden pressure predominate. Furthermore, these studies have been restricted to particular variations which have allowed the resulting simplification to be exploited.

Since our earth is a spherical body having finite dimension, the elastic waves generated must receive the effect of the boundaries. Naturally, this phenomenon leads us to the investigation of boundary waves or surface waves, i.e. the waves, which are confined to some surface during their propagation. Although much literature is available on the propagation of surface waves, i.e. Rayleigh waves, Love waves and Stoneley waves etc., in a non-homogeneous elastic media in the monographs of Stoneley [1], Bullen [2], Ewing et al. [3], Hunters [4] and Jeffreys [5], but very little literature is available on the propagation of another type of surface wave called torsional surface wave whose amplitudes decay exponentially with distance from the free surface and horizontally polarized but give a twist to the medium when it propagates. These waves often propagate during the earthquakes and become responsible to some extent for the destruction on the earth crustal layer. Therefore seismologists have started taking a keen interest on the propagation of torsional surface waves in different geo-media.

Sezawa [6] studied the dispersion of elastic waves propagated on curved surfaces. The transmission of elastic waves through a stratified solid medium was studied by Thomson [7]. Haskell [8] studied the dispersion of surface waves in multilayered media. Biot [9] studied the influence of gravity on Rayleigh waves, assuming the force of gravity to create a type of initial stress of hydrostatic nature and the medium to be incompressible.

Propagation of Love waves in a non-homogeneous stratum of finite depth sandwiched between two semi infinite isotropic media was studied earlier by Sinha [10]. Wave propagation in a thin two-layered laminated medium with couple under initial stress was studied by Roy [11]. Datta [12] studied the effect of gravity on Rayleigh wave propagation in a homogeneous, isotropic elastic solid medium. Effects of irregularities on the propagation of guided SH waves were studied by Chattopadhyay et al. [13]. Goda [14] studied the effect of non-homogeneity and anisotropy on Stoneley waves. Gupta et al. [15] investigated the influence of linearly varying density and rigidity on torsional surface waves in an inhomogeneous crustal layer.

Some of the recent notable works on propagation of torsional waves in various media with different geometries were done by Georgiadis [16], Dey and Sarkar [17], Selim [18], Ozturk and Akbbarov [19] and Gupta et al. [20]. Torsional wave propagation in a thin rectangular domain using the asymptotic approach was studied by Davini et al. [21], whereas Dey et al. [22] investigated the torsional surface wave in an elastic layer with void pores.

Recently, Sethi and Gupta [23] investigated surface waves in homogeneous visco-elastic media of higher order under the influence of surface stresses.

In the present problem, we have considered the propagation of torsional surface waves in a nonhomogeneous half-space with an irregular free surface. The expression for displacement and dispersion equation is found in the closed form. The dispersion curves are depicted by means of graphs for different values of non-homogeneity parameters and irregularity parameter. The influence of non-homogeneity parameters and irregularity on the dimensionless phase velocity has been studied.

Here, the non-homogeneity in the half-space can be taken as

$$
\begin{aligned}
\mu & =\frac{\mu_{0}}{1+a z}, \\
\rho & =\frac{\rho_{0}}{1+b z}, \quad \text { where } \quad a>0, \quad b>0 .
\end{aligned}
$$

The equation of irregularity has been taken as

$$
z=\varepsilon F(r), \quad \text { where } \quad F(r)=\left\{m^{2}\left(1-\frac{r^{2}}{m^{2}}\right)\right\}, \quad|r| \leq m \quad \text { and } \quad 0, \quad|r| \geq m
$$

where $a$ and $b$ are constants and having the dimensions that are inverse of length. 


\section{Formulations of problem and solution}

We consider the propagation of torsional surface waves in a non-homogeneous half-space with an irregular free surface. For the study of torsional waves, a cylindrical coordinate system is used.

The origin of the cylindrical coordinate system $(r, \theta, z)$ is located at the interface of half-space and the origin is chosen at the middle point of the irregularity. We chose the $z$-axis vertically downwards and the $r$-axis act horizontally along the interface. The following variation in the rigidity and the mass density are taken into account as shown in Fig.1.

$$
\begin{aligned}
& \mu=\frac{\mu_{0}}{1+a z}, \\
& \rho=\frac{\rho_{0}}{1+b z}, \quad \text { where } \quad a>0, \quad b>0 .
\end{aligned}
$$

where $a$, and $b$ are constants having dimensions that are inverse of length.

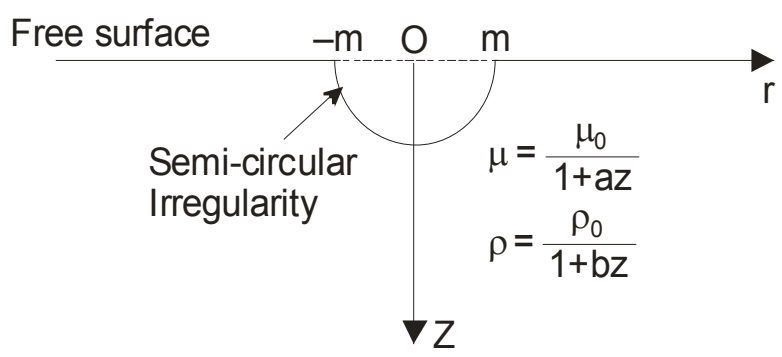

Fig.1. Geometry of problem.

In the present problem, we assume that the free surface may be taken as $z=\varepsilon F(r)$, where $\varepsilon(<<1)$ be the small quantity so that its higher powers may be neglected. We assume $m$ be the radius of semi-circular irregularity and $2 m$ be the span of irregularity.

The equation of irregularity has been taken as

$$
z=\varepsilon F(r), \quad \text { where } \quad F(r)=\left\{m \sqrt{\left(1-\frac{r^{2}}{m^{2}}\right)},|r| \leq m \text { and } 0,|r| \geq m\right\} .
$$

The dynamic equations of motion are

$$
\begin{aligned}
& \frac{\partial \sigma_{r r}}{\partial r}+\frac{1}{r} \frac{\partial \sigma_{r \theta}}{\partial \theta}+\frac{\partial \sigma_{r z}}{\partial z}+\frac{\sigma_{r r}-\sigma_{\theta \theta}}{r}=\rho \frac{\partial^{2} u}{\partial t^{2}}, \\
& \frac{\partial \sigma_{r \theta}}{\partial r}+\frac{1}{r} \frac{\partial \sigma_{\theta \theta}}{\partial \theta}+\frac{\partial \sigma_{\theta z}}{\partial z}+\frac{2 \sigma_{r \theta}}{r}=\rho \frac{\partial^{2} v}{\partial t^{2}}, \\
& \frac{\partial \sigma_{r z}}{\partial r}+\frac{1}{r} \frac{\partial \sigma_{\theta z}}{\partial \theta}+\frac{\partial \sigma_{z z}}{\partial z}+\frac{\sigma_{r z}}{r}=\rho \frac{\partial^{2} w}{\partial t^{2}}
\end{aligned}
$$


where $\sigma_{r r}, \sigma_{\theta \theta}, \sigma_{z z}, \sigma_{r z}, \sigma_{r \theta}$, and $\sigma_{\theta z}$, are the respective stress components, and $u, v$, and $\mathrm{w}$ are the respective displacement components. Now, the stress-strain are given by

$$
\begin{aligned}
& \sigma_{r r}=\lambda \Omega+2 \mu e_{r r}, \quad \sigma_{\theta \theta}=\lambda \Omega+2 \mu e_{\theta \theta}, \\
& \sigma_{z z}=\lambda \Omega+2 \mu e_{z z}, \quad \sigma_{r \theta}=2 \mu e_{r \theta}, \\
& \sigma_{r z}=2 \mu e_{r z}, \quad \sigma_{\theta z}=2 \mu e_{\theta z},
\end{aligned}
$$

where $\lambda$ and $\mu$ are Lame's constant, and $\Omega=\left(\frac{\partial u}{\partial r}+\frac{1}{r} \frac{\partial v}{\partial \theta}+\frac{u}{r}+\frac{\partial w}{\partial z}\right)$ denotes the dilatation. The straindisplacement relations are

$$
\begin{aligned}
& e_{r r}=\frac{1}{2} \frac{\partial u}{\partial r}, \quad e_{\theta \theta}=\frac{1}{2}\left(\frac{1}{r} \frac{\partial v}{\partial \theta}+\frac{u}{r}\right), \quad e_{z z}=\frac{1}{2} \frac{\partial w}{\partial z}, \\
& e_{r \theta}=\frac{1}{2}\left(\frac{1}{r} \frac{\partial u}{\partial \theta}+\frac{\partial v}{\partial r}+\frac{v}{r}\right), \quad e_{\theta z}=\frac{1}{2}\left(\frac{\partial v}{\partial z}+\frac{1}{r} \frac{\partial w}{\partial \theta}\right), \\
& e_{z r}=\frac{1}{2}\left(\frac{\partial w}{\partial r}+\frac{\partial u}{\partial z}\right) .
\end{aligned}
$$

The torsional wave is characterized by the displacements

$$
u=0, \quad w=0, \quad v=v(r, z, t) .
$$

Now, considering Eqs (2.4)-(2.6), the dynamical equations of motion for torsional surface waves propagating in the radial direction can be written as

$$
\frac{\partial \sigma_{r \theta}}{\partial r}+\frac{\partial \sigma_{z \theta}}{\partial z}+\frac{2 \sigma_{r \theta}}{r}=\rho(z) \frac{\partial^{2} v}{\partial t^{2}}
$$

where $v(r, z, t)$ is the displacement along the $\theta$ (azimuthal) direction, and $r$ is the radial coordinates. The stresses are related to the displacement component by

$$
\sigma_{r \theta}=\mu(z)\left(\frac{\partial v}{\partial r}-\frac{v}{r}\right), \quad \sigma_{z \theta}=\mu(z)\left(\frac{\partial v}{\partial z}\right)
$$

Using Eq.(2.8), Eq.(2.7) takes the form

$$
\mu(z)\left(\frac{\partial^{2}}{\partial r^{2}}+\frac{1}{r} \frac{\partial}{\partial r}-\frac{1}{r^{2}}\right) v+\frac{\partial}{\partial z}\left(\mu(z) \frac{\partial v}{\partial z}\right)=\rho(z) \frac{\partial^{2} v}{\partial t^{2}} .
$$

Harmonic wave solution of Eq.(2.9) is of the form

$$
v=V(z) J_{l}(\mathrm{Kr}) \exp (i \omega t)
$$


where $\omega$ is the angular frequency, $K$ is the angular wave number, and $J_{l}(K r)$ is the first order Bessel function of first kind.

Substituting Eq.(2.10) in Eq.(2.9) and using Eq.(2.2), we have

$$
\frac{d^{2} V}{d z^{2}}-\frac{a}{1+a z} \frac{d V}{d z}-K^{2}\left(1-\frac{c^{2}}{c_{0}^{2}} \frac{1+a z}{1+b z}\right) V(z)=0
$$

where $c=\omega / K$ is the torsional wave velocity and $c_{0}^{2}=\mu_{0} / \rho_{0}$.

Now, substituting $V(z)=\frac{g(z)}{(1+a z)^{-1 / 2}}$ into Eq.(2.11) to eliminate the term $\frac{d V}{d z}$, we have

$$
\frac{d^{2} g(z)}{d z^{2}}+\left[-\frac{3 a^{2}}{4(1+a z)^{2}}-K^{2}\left(1-\frac{c^{2}}{c_{0}^{2}} \frac{1+a z}{1+b z}\right)\right] g(z)=0 .
$$

Introducing the dimensions quantities

$$
\gamma=\sqrt{1-\frac{c^{2}}{c_{0}^{2}} \frac{a}{b}}
$$

and

$$
\eta=\frac{2 \gamma K(1+a z)}{a}
$$

in Eq.(2.12), we have

$$
\frac{d^{2} g}{d z^{2}}+\left(-\frac{1}{4}-\frac{3}{4 \eta^{2}}+\frac{R}{\eta}\right) g(\eta)=0, \quad R=\frac{K a}{2 \gamma} \frac{c^{2}}{c_{0}^{2}}\left(\frac{1}{b^{2}}\left(1-\frac{b}{a}\right)\right)
$$

which is the well known Whittaker's equation [24].

The solution of Whittaker's Eqs (2.13) is given by

$$
g(\eta)=A W_{R, 1}(\eta)+B W_{-R, l}(-\eta)
$$

where $W_{R, I}(\eta)$ and $W_{-R, 1}(-\eta)$ are Whittaker functions.

So for the heterogeneous half -space, solution should vanish at $z \rightarrow \infty$ i.e. $\eta \rightarrow \infty$. So in view of the above condition the solution may be taken as

$$
g(\eta)=A W_{R, 1}(\eta)
$$

Hence, the displacement for the torsional wave in the heterogeneous half-space is

$$
v=A W_{R, I}(\eta)(1+a z)^{I / 2} J_{l}(K r) e^{i \omega t}, \quad \eta=\frac{2 \gamma K}{a}(1+a z) .
$$




\section{Boundary condition}

In the absence of any external body forces in the boundary, the surface of the medium is kept stress free at $z=\varepsilon F(r)$ and also, we assume that the displacement at $r=0$ is finite.

So, the boundary condition may be taken as

$$
\mu(z) \frac{d v}{d z}=0 \quad \text { at } \quad z=\varepsilon F(r) .
$$

Expanding Whittaker's function up to linear term and using the boundary condition (3.1), one find the wave velocity equation from Eq.(2.14) as under

$$
\left[\frac{4 \gamma K(1+a \varepsilon F(r))}{a}\left\{\left(-0.5+\frac{R}{\eta}\right)\left(1-\frac{(R+0.5)^{2}}{\eta}\right)+\frac{(R+0.5)^{2}}{\eta^{2}}\right\}+\left(1-\frac{(R+0.5)^{2}}{\eta}\right)\right] e^{-\eta / 2} \eta^{R}=0 .
$$

After simplification we have

$$
\frac{c}{c_{0}}=\sqrt{\frac{b}{a}}
$$

or

$$
\begin{aligned}
& (R+0.5)^{2}\left[\frac{2 \gamma K}{a}\left\{1+a m \varepsilon \sqrt{1-\frac{r^{2}}{m^{2}}}\right\}-2 R+1\right]+ \\
& +\frac{2 \gamma K}{a}\left\{1+a m \varepsilon \sqrt{1-\frac{r^{2}}{m^{2}}}\right\}\left[2 R+1-\frac{2 \gamma K}{a}\left\{1+a m \varepsilon \sqrt{1-\frac{r^{2}}{m^{2}}}\right\}\right]=0 .
\end{aligned}
$$

\section{Particular cases}

Case I: When $\varepsilon \rightarrow 0$, i.e. in the absence of irregularity in the half-space, Eq.(3.2) reduces to

$$
(R+0.5)^{2}\left[\frac{2 \gamma K}{a}-2 R+1\right]+\frac{2 \gamma K}{a}\left[2 R+1-\frac{2 \gamma K}{a}\right]=0
$$

which is the dispersion equation of torsional surface waves in a non-homogeneous elastic half-space.

Case II: When $a=b, \varepsilon \rightarrow 0$, i.e., both rigidity and density components in half-space having similar variation and in the absence of irregularity in the half-space, Eq.(3.2) reduces to

$$
\frac{c^{4}}{c_{0}^{4}}+\frac{c^{2}}{c_{0}^{2}}\left[-2+\frac{33}{64} \frac{a^{2}}{K^{2}}\right]+1-\frac{33}{64} \frac{a^{2}}{K^{2}}+\frac{a^{2}}{256^{4}}=0 .
$$

\section{Numerical computations and discussion}

In order to demonstrate the effect of irregularity and dispersion of non-homogeneities on the propagation of torsional surface wave in non-homogeneous isotropic elastic half-space, phase velocity is 
calculated numerically with the help of MATLAB from Eq.(3.2). Effects of non-homogeneity parameters and irregularity parameter on torsional surface waves in a non-homogeneous isotropic elastic half-space is discussed in the following way by means of graphs.

Figure 2 shows the effect of the irregularity parameter $\varepsilon$ on the dimensionless phase velocity $\left(c / c_{0}\right)$ of torsional surface waves. Following observations and effects are obtained under the above considered values. (1a) For various values of the non-homogeneity factor in rigidity, i.e. $K / a$ and fixed value of nonhomogeneity factor in density of the half space, i.e. $K / b=2.0$, the dimensionless phase velocity $\left(c / c_{0}\right)$ of torsional surface waves increases, as the value of the irregularity parameter $\varepsilon$ increases from 0.2 to 0.4 , in comparison with the case of $\varepsilon=0.0$.

(1b) For a particular value of the non-homogeneity factor in density of the half space, i.e. $K / b=2.0$, the dimensionless phase velocity $\left(c / c_{0}\right)$ decreases as the non-homogeneity factor in rigidity, i.e. $K / a$, increases from 4.0 to 7.0 .

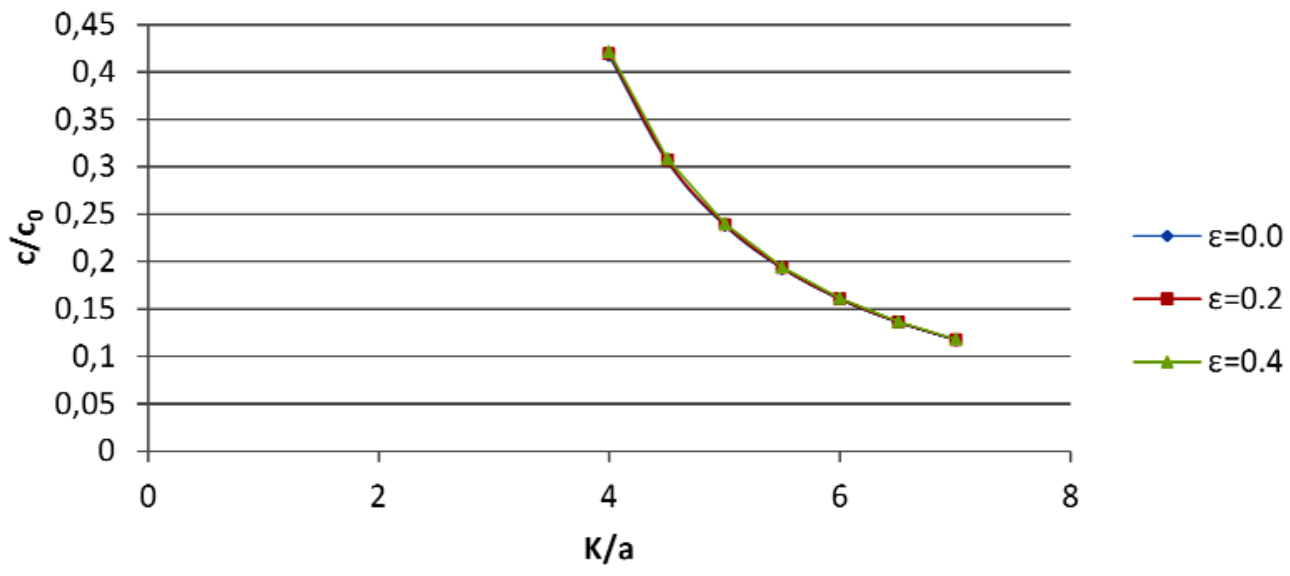

Fig.2. Variation of dimensionless phase velocity $c / c_{0}$ against dimensionless $K / a$ evaluated from Eq.(3.2) demonstrating the influence of non-negative values of irregularity parameter $\varepsilon$ for $K / b=2.0, K m=0.2$, $r / m=0.1$.

Figure 3 gives the same results for negative values of the irregularity parameter $\varepsilon$.

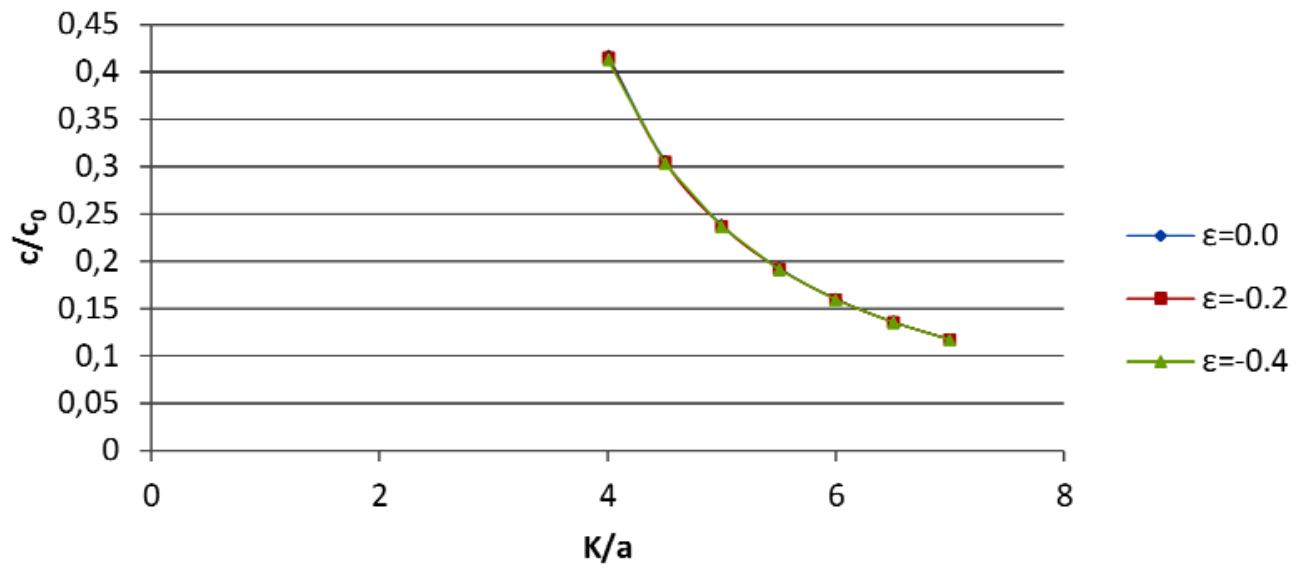

Fig.3. Variation of dimensionless phase velocity $c / c_{0}$ against dimensionless $K / a$ evaluated from Eq.(3.2) demonstrating the influence of non-positive values of irregularity parameter $\varepsilon$ for $K / b=2.0, K m=$ $0.2, r / m=0.1$. 
Figure 4 shows the effect of the irregularity parameter $\varepsilon$ on the dimensionless phase velocity $\left(c / c_{0}\right)$ of torsional surface waves. Following observations and effects are obtained under the above considered values.

(2a) For various values of the non-homogeneity factor in density, i.e. $K / b$ and fixed value of nonhomogeneity in rigidity of the half space, i.e. $K / a=3.0$, the dimensionless phase velocity $\left(c / c_{0}\right)$ of torsional surface waves increases, as the value of irregularity parameter $\varepsilon$ increases from 0.2 to 0.4 , on comparison with the case of $\varepsilon=0.0$.

(2b) For particular value of non-homogeneity factor in rigidity of half space i.e. $K / a=3.0$, the dimensionless phase velocity $\left(c / c_{0}\right)$ decreases as the non-homogeneity factor in density, i.e. $K / b$, increases from 0.2 to 1.0 .

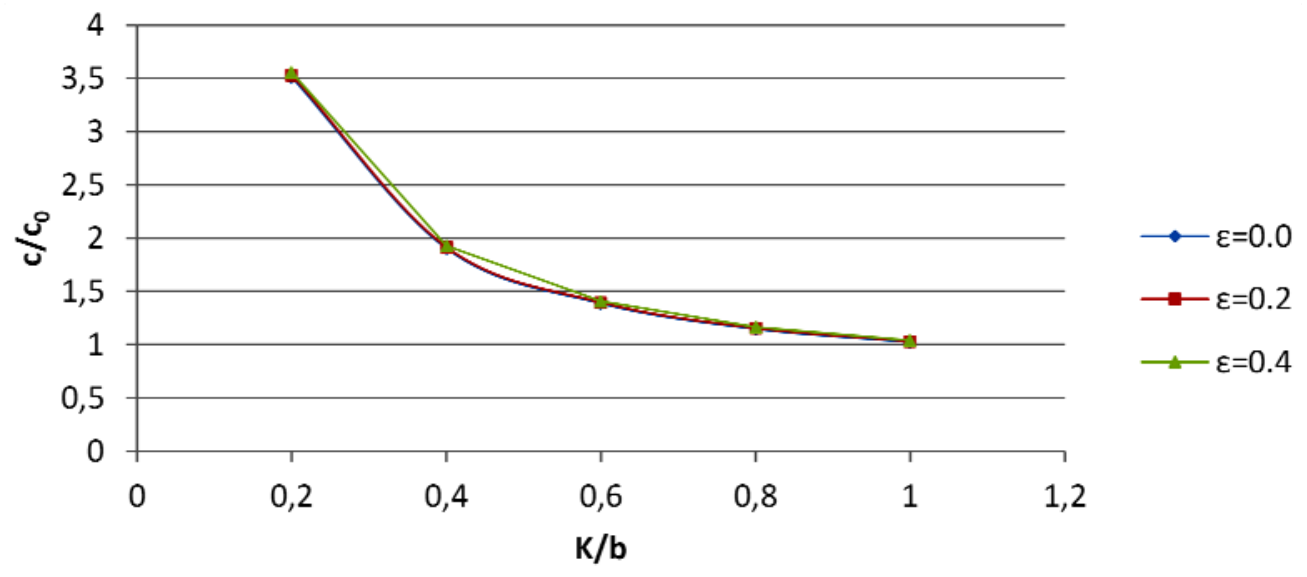

Fig.4. Variation of dimensionless phase velocity $c / c_{0}$ against dimensionless $K / b$ evaluated from Eq.(3.2) demonstrating the influence of non-negative values of irregularity parameter $\varepsilon$ for $K / a=3.0, \mathrm{Km}=$ $0.2, r / m=0.1$.

Figure 5 gives the same results for negative values of the irregularity parameter $\varepsilon$.

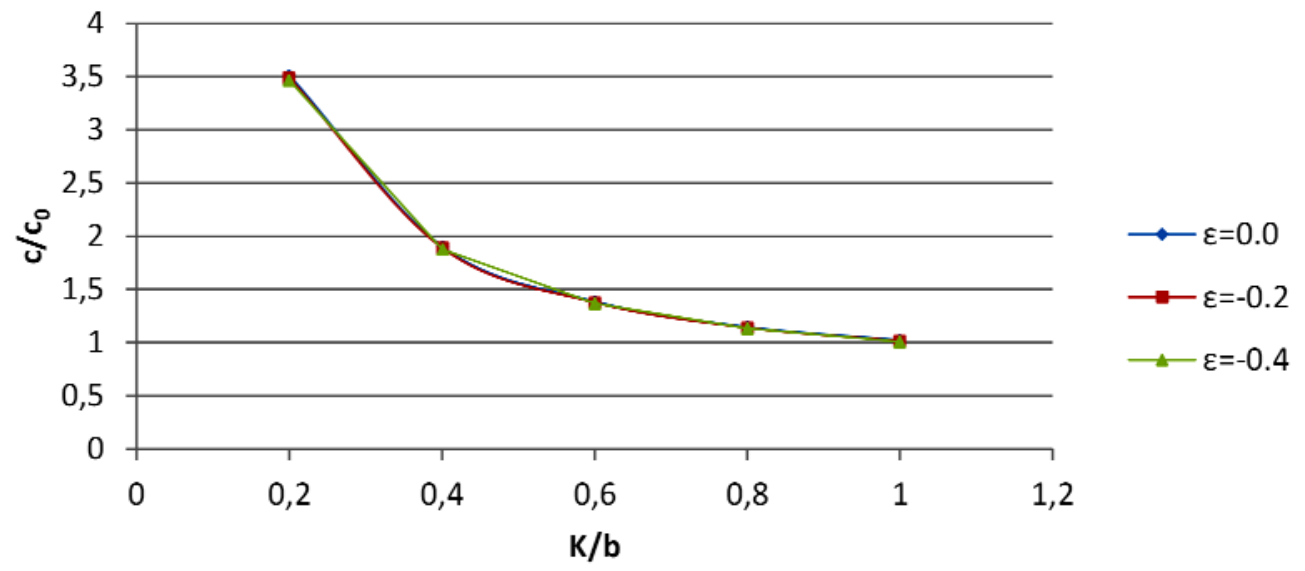

Fig.5. Variation of dimensionless phase velocity $c / c_{0}$ against dimensionless $K / a$ evaluated from Eq.(3.2) demonstrating the influence of non-positive values of irregularity parameter $\varepsilon$ for $K / a=3.0, K m=$ $0.2, r / m=0.1$.

When the non-homogeneities in both rigidity and density are same as shown in Fig.6, it is observed that the dimensionless phase velocity $\left(c / c_{0}\right)$ of torsional surface waves increases, as the value of the nonhomogeneity factor in semi-infinite media, i.e. $K / a$, increases. 


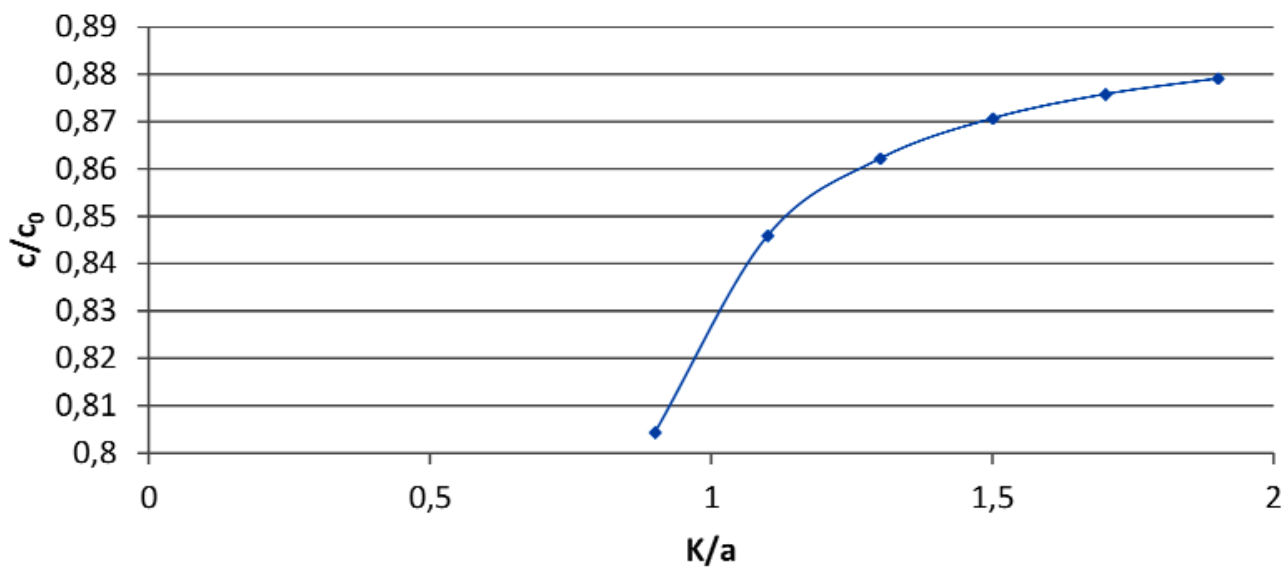

Fig.6. Variation of dimensionless phase velocity $c / c_{0}$ against dimensionless $K / a$ evaluated from Eq.(3.2) in the absence of irregularity parameter $\varepsilon$ for $K / a=3.0, K m=0.2, r / m=0.1$.

\section{Conclusions}

Here, we have investigated the propagation of torsional surface waves in a non-homogeneous isotropic elastic half-space, with free irregular surface. A closed form solution has been derived for the displacement in the elastic half-space. By using the asymptotic expansion of Whittaker's function we have derived the wave velocity equation for the torsional surface waves in compact form. Dimensionless phase velocity is calculated numerically with the help of MATLAB. The effects of various dimensionless nonhomogeneity parameters and the irregularity parameter on the dimensionless phase velocity $\left(c / c_{0}\right)$ have been shown graphically. We make the following conclusions

1 The dimensionless phase velocity $\left(c / c_{0}\right)$ of torsional surface waves increases, as the value of irregularity parameter $\varepsilon$ increases, in comparison with the value of the dimensionless phase velocity $\left(c / c_{0}\right)$ in the absence of irregularity. Thus, the irregularity parameter has a prominent effect on the propagation of torsional surface waves in a heterogeneous Earth medium.

2 The dimensionless phase velocity $\left(c / c_{0}\right)$ of torsional surface waves decreases, as the value of the nonhomogeneity parameters in rigidity and density of the irregular half-space, i.e. $K / a$ and $K / b$, increases. Thus, the non-homogeneity parameters have a prominent effect on the propagation of torsional surface waves in a heterogeneous Earth medium.

3 In case of same type of non-homogeneity in rigidity and density, the dimensionless phase velocity $\left(c / c_{0}\right)$ of torsional surface waves increases, as the value of non-homogeneity factor in semi-infinite media, i.e. $K / a$, increases.

\section{Nomenclature}

$$
\begin{aligned}
a, \text { and } b & - \text { constants having dimensions that are inverse of length } \\
c & - \text { torsional wave velocity } \\
e_{r r}, e_{\theta \theta}, e_{z z}, e_{r z}, & - \text { strain components } \\
e_{r \theta} \text { and } e_{\theta z} & \\
K & - \text { angular wave number } \\
m & - \text { radius of semi-circular irregularity } \\
2 m & - \text { span of irregularity } \\
v(r, z, t) & - \text { displacement along the azimuthal direction } \\
\alpha, \mu & - \text { elastic constants } \\
\varepsilon & - \text { irregularity }
\end{aligned}
$$




$$
\begin{aligned}
& \rho-\text { density of the material medium } \\
& \sigma_{r r}, \sigma_{\theta \theta}, \sigma_{z z}, \sigma_{r z}, \quad \text { - stress components } \\
& \sigma_{r \theta} \text { and } \sigma_{\theta z} \\
& \Omega \text { - dilatation } \\
& \omega-\text { angular frequency } \\
& \nabla^{2}=\frac{\partial^{2}}{\partial x^{2}}+\frac{\partial^{2}}{\partial z^{2}}-\text { Laplacian operator }
\end{aligned}
$$

\section{References}

[1] Stoneley R. (1924): Waves at the surface of separation of two solids. - Proc. R. Soc. A 806. pp.416-428.

[2] Bullen K.E. (1965). Theory of Seismology. - Cambridge University Press.

[3] Ewing W.M., Jardetzky W.S. and Press F. (1957): Elastic Waves in Layered Media. - New York: McGraw-Hill.

[4] Hunter S.C. (1970). Viscoelastic Waves. - Progress in Solid Mechanics, I. (ed: Sneddon IN and Hill R) Cambridge University Press.

[5] Jeffreys H. (1970): The Earth. - Cambridge University Press.

[6] Sezawa K. (1927): Dispersion of elastic waves propagated on the surface of stratified bodies and on curved surfaces. - Bull. Earthq. Res. Inst. Tokyo, vol.3. pp.1-18.

[7] Thomson W. (1950): Transmission of elastic waves through a stratified solid medium. - J. Appl. Phys., vol.21, pp.89-93.

[8] Haskell N.A. (1953): The dispersion of surface waves in multilayered media. - Bull. Seis. Soc. Amer., vol.43. pp.17-34.

[9] Biot M.A. (1965): Mechanics of Incremental Deformations. - J. Willy.

[10] Sinha N (1967): Propagation of Love waves in a non-homogeneous stratum of finite depth sandwiched between two semi-infinite isotropic media. - Pure Applied Geophysics, vol.67. pp.65-70.

[11] Roy P.P. (1984): Wave propagation in a thin two layered medium with stress couples under initial stresses. - Acta Mechanics, vol.54, pp.1-21.

[12] Datta B.K. (1986): Some observation on interactions of Rayleigh waves in an elastic solid medium with the gravity field. - Rev. Roumaine Sci. Tech. Ser. Mec. Appl., vol.31. pp.369-374.

[13]Chattopadhyay A., Chakraborty M. and Pal A.K. (1983): Effects of irregularity on the propagation of guided SH waves. - Jr. de Mecanique Theo. et Appl., vol.2, No.2. pp.215-225.

[14] Goda M.A. (1992): The effect of inhomogeneity and anisotropy on Stoneley waves. - Acta Mech., vol.93, No.1-4. pp.89-98.

[15] Gupta S., Vishwakarma S.K., Majhi D.K. and Kundu S. (2012): Influence of linearly varying density and rigidity on torsional waves in inhomogeneous crustal layer. - Appl. Math. Mech.-Engl. Ed., vol.33, No.10, pp.1239-1252.

[16] Georgiadis H.G., Vardaulakis I. and lykotrafitis G. (2000): Torsional surface wave in gradient-elastic half-space. Wave Motion, vol.31, No.4, pp.333-348.

[17] Dey S. and Sarkar M.G. (2002): Torsional surface waves in an initially stressed anisotropic porous medium. - J. Eng. Mech., vol.128, No.2, pp.184-189.

[18] Selim M.M. (2007): Propagation of torsional surface wave in heterogeneous half-space with irregular free surface. - Appl. Math. Sci., vol.1, No.29-32, pp.1429-1437.

[19] Ozturk A. and Akbbarov S.D. (2009): Torsional wave propagation in a pre-stressed circular cylinder embedded in a pre-stressed elastic medium. - Appl. Math. Model., vol.33, pp.3636-3649. 
[20] Gupta S., Majhi D.K., Kundu S. and Vishwakarma S.K. (2012): Propagation of torsional surface waves in a homogeneous layer of finite thickness over an initially stressed heterogeneous half-space. - Appl. Math. Comput., vol.218, pp.5655-5664.

[21] Davini C., Paroni R. and Puntle E. (2008): An asymptotic approach to the torsional problem in thin rectangular domains. - Meccanica, vol.43, No.4, pp.429-435.

[22] Dey S., Gupta S., Gupta A.K., Kar S.K. and De P.K. (2003): Propagation of torsional surface waves in an elastic layer with void pores over an elastic half-space with void pores. - Tamkang J. Sci. Eng., vol.6, No.4, pp.241-249.

[23] Sethi M., Gupta K.C., Rani M. and Vasudeva A. (2013): Surface waves in homogeneous viscoelastic media of higher order under the influence of surface stresses. - J. Mech. Behav. Mater., vol.22, No.5-6, pp.185-191.

[27] Whittaker E.T. and Watson G.N. (1990): A Course in Modern Analysis. - $4^{\text {th }}$ edn, Cambridge: Cambridge University Press.

Received: December 16, 2016

Revised: September 12, 2018 